\title{
Ultra-Low-Power Multi-function Data Collection System Based on MSP430 and uC/OS-II
}

\author{
CHEN Song ${ }^{1,2}$ \\ ${ }^{1}$ High magnetic field laboratory, Chinese academy of \\ sciences, Hefei 230031, China \\ ${ }^{2}$ University of Science and Technology of China, Hefei \\ 230026, China \\ E-mail: gengyun97@163.com
}

WU Zhong-cheng

High magnetic field laboratory, Chinese academy of sciences, Hefei 230031, China

E-mail: zcwu@iim.ac.cn

\begin{abstract}
This data collection system is based on MSP430, which is an ultra-low power 16-bit micro-controller. Multi-task application program is designed in $\mathrm{uC}$ /OSII, which is real-time operating system. It can handle a variety of meter signal and communicate with concentrators. This system has a good realtime and stability, applicable to transcribing of residential energy consumption.
\end{abstract} power

Keywords-data collection; MSP430; uC/OS-II; ultra-low

\section{INTRODUCTION}

Signals of the residential district meters possibly have many kinds of forms, if the collector can only receive pulse signal or simulated signal, the application is restricted. This article designs one kind of collector, which may process many kinds of signals, including the impulse signal, the electric current signal, the digital signal transmitted by the RS-232 connection, the digital signal transmitted by NRF905 wireless module. According to the user's need, signal processing modules will be fixed in this system which may apply in many kinds of situations and many kinds of meters' data acquisition.

MSP430 micro-controller is used in this design, so the power- consumption is low, the function is complete, and the operating speed is fast. $\mu \mathrm{C} / \mathrm{OS}-\mathrm{II}$ is used as the embedded operating system, to realize the multi-tasks. It is specially the real-time multi-duty operating system essence which is designed for the micro-controller system and the software development. It's a background procedure which carries out first after micro-controllers start. The source code has been publicized, perspicuous, and the probability is good. The essence belongs to the grab type, and it may manage 60 duties at most.
Supported by Anhui University Natural Science Research Project funding (KJ2016A156); Supported by National Natural Science Foundation of China (61071001).

\author{
WU Zi-heng ${ }^{1,2}$ \\ ${ }^{1}$ High magnetic field laboratory, Chinese academy of \\ sciences, Hefei 230031, China \\ ${ }^{2}$ University of Science and Technology of China, Hefei \\ 230026, China \\ E-mail: 282604514@qq.com
}

LV Hong

College of Mechanical and Electrical Engineering, Anhui Jianzhu University

Hefei 230601, China

\section{HARDWARE Design OF COLLECTION SYSTEM}

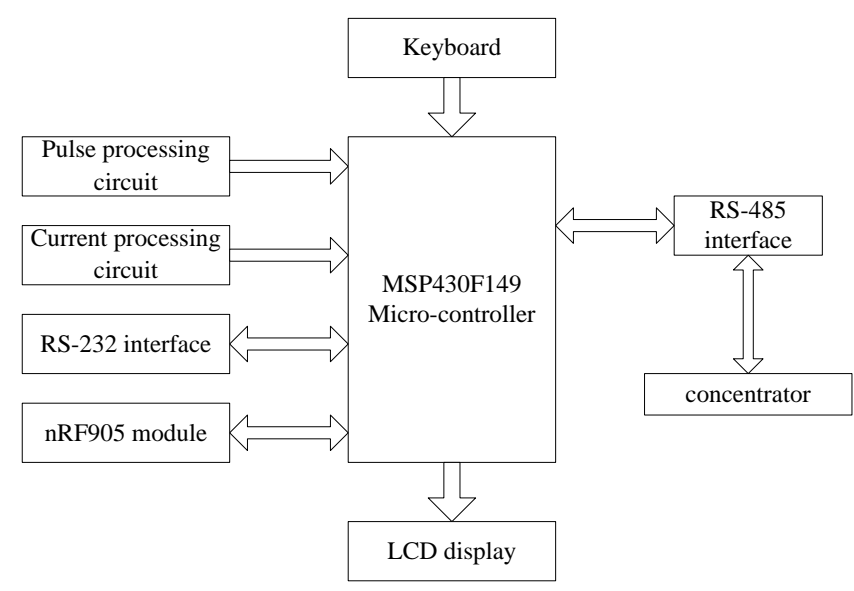

Fig. 1. Data collection system's structure

MSP430F149 micro-controller is used as the core processor, to realize energy-consumption data collection and the data memory. Energy-consumption value is displayed on LCD. The collector can communicate with concentrator in the residential district through the RS-485 connection. Users may use the keys to establish the collection parameter and the correspondence parameter. Collection system's structure is shown in Fig. 1.

\section{A. Pulse signal processing circuit}

Pulse processing electric circuit is shown in Fig. 2. The input signal is sent to a second-order low pass filter, whose function is removing high frequency interference in the impulse signal. The normal signal impulse is smaller than 10 $\mathrm{kHz}$, so the cut-off frequency of filter is $10 \mathrm{kHz}$. From this we can conclude the resistance value is $1.58 \mathrm{k} \Omega$, and the capacitance value is the $0.01 \mathrm{uF}$. Electro-optic coupler's function is isolating the meter's circuit and the micro- 
controller circuit, in case that the meter's electric current is oversized to damage micro-controller circuit. Schmitt trigger's function is removing the pulse jitter.

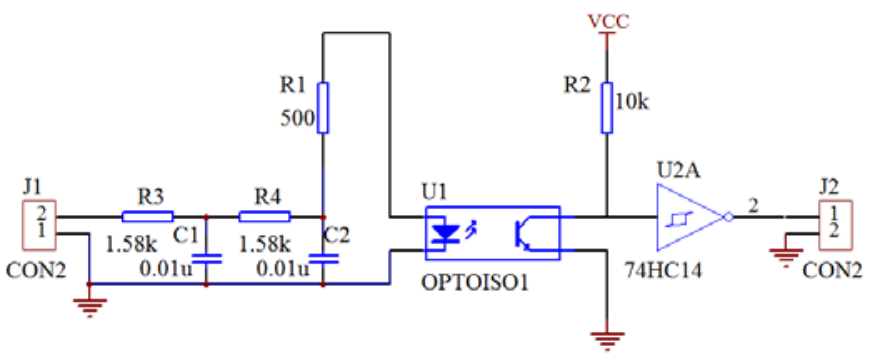

Fig. 2. Pulse signal processing circuit

\section{B. RS-232 interface}

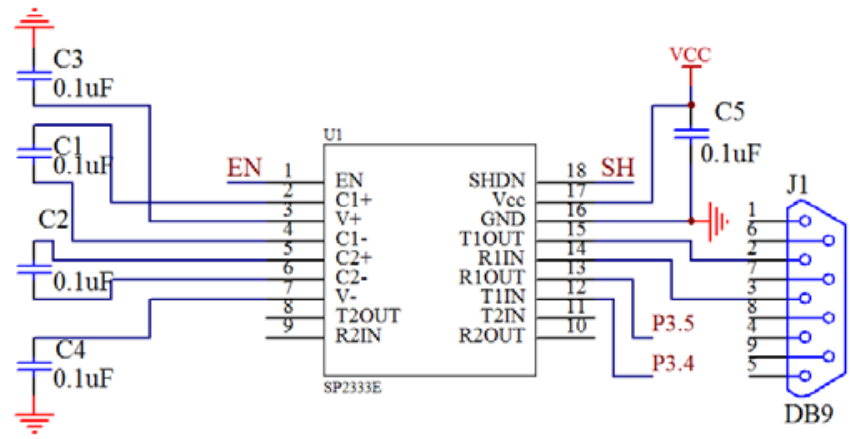

Fig. 3. RS-232 interface circuit

Certain sensor surveying electric quantity communicates with the micro-controller through RS-232 interface. If the request of the transmission's speed and distance is not high, the RS-232 connection can be used and make the structure simple, performance-to-price ratio high. SP3222E is selected, which has the following characteristic: It has a low-power shutdown mode and the supply current falls to $1 \mathrm{uA}$ during shutdown; Uses $+3 \mathrm{~V} \sim+5.5 \mathrm{~V}$ to supply power; the temperature range is $0 \sim 70^{\circ} \mathrm{C}$; Internal integrates two RS-232 drivers and receivers; May withstand $\pm 15 \mathrm{kV}$ human body electric discharge. T1IN port and R1OUT port are connected to UART0 of micro-controller. T1OUT port and R1IN port are connected to meter circuit. Electric circuit connection is shown in Fig. 3.

\section{Current signal processing circuit}

Certain meter circuit outputs $4 \sim 20 \mathrm{~mA}$ electric current signal, in this design the electric current signal is changed to voltage signal which is sent to ADC, and to micro-controller for further processing.

AD620 instrumentation amplifier, with high accuracy and low offset voltage is used. It is easy to use, as the multiple is established only by a resistance outside. Its powerconsumption is very low, and max supply current is $1.3 \mathrm{~mA}$.

$\pm 5 \mathrm{~V}$ supplies power is provided for AD620. The AD620’s 2nd pin meets $1 \mathrm{~V}$ reference voltage. $200 \mathrm{k} \Omega$ resistance is used to set enlargement factor of amplifier. The voltage signal inputs 3rd pin, after enlargement the voltage outputs from 6th pin, then inputs ADC of micro-controller. Electric circuit connection is shown in Fig. 4. 4 20mA electric current signal is added to $250 \Omega$ resistance, transformed to $1 \sim 5 \mathrm{~V}$ voltage signal, and results in $0 \sim 4 \mathrm{~V}$ with the reference voltage cancellation. $200 \mathrm{k} \Omega$ resistance is chosen, and the gain equation is $\left(\mathrm{G}=1+49.4 \mathrm{k} \Omega / \mathrm{R}_{\mathrm{G}}\right)$, so that $\mathrm{G}=1.247$, and output voltage of the amplifier is $0 \sim 4.988 \mathrm{~V}$.

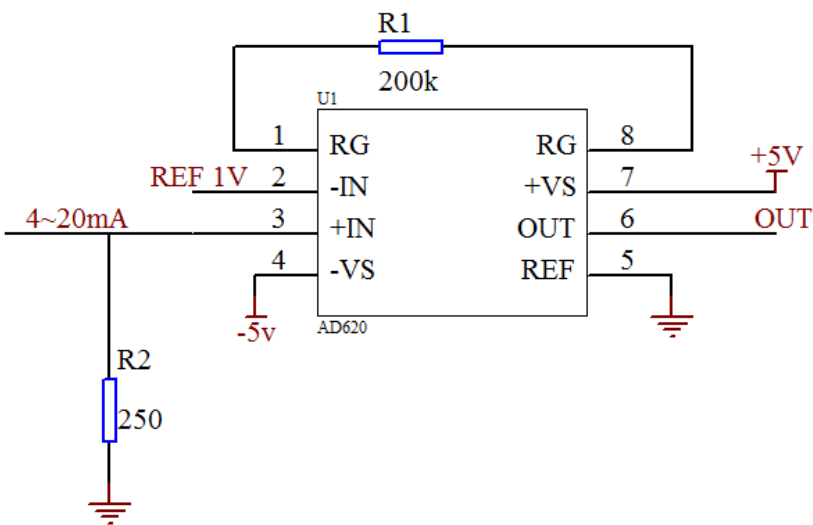

Fig. 4. Current signal processing circuit

\section{D. nRF905 module}

nRF905 and MSP430 circuit diagram is shown in Fig. 5, MOSI, MISO, SCK are connected with micro-controller SPI pins, and CSN, TX_EN, TRX_CE, PWR_UP with IO pins; $\mathrm{DR}, \mathrm{CD}$, AM meet $\mathrm{P} 2$ pins of MSP430 which are multiplexing interruption ports, to use the interruption to inform MSP430 promptly. nRF905 may establish two kinds of working modes and two kinds of energy-conservation modes. Energyconservation mode includes close-down mode and idle mode. In the close-down mode, the operating current is smallest, generally $2.5 \mathrm{uA}$. In the close-down mode, it maintains the content in the configuration-register, but will not accept or the transmit data. In the working mode, the electric current is $12.5 \mathrm{~mA}$ generally. 


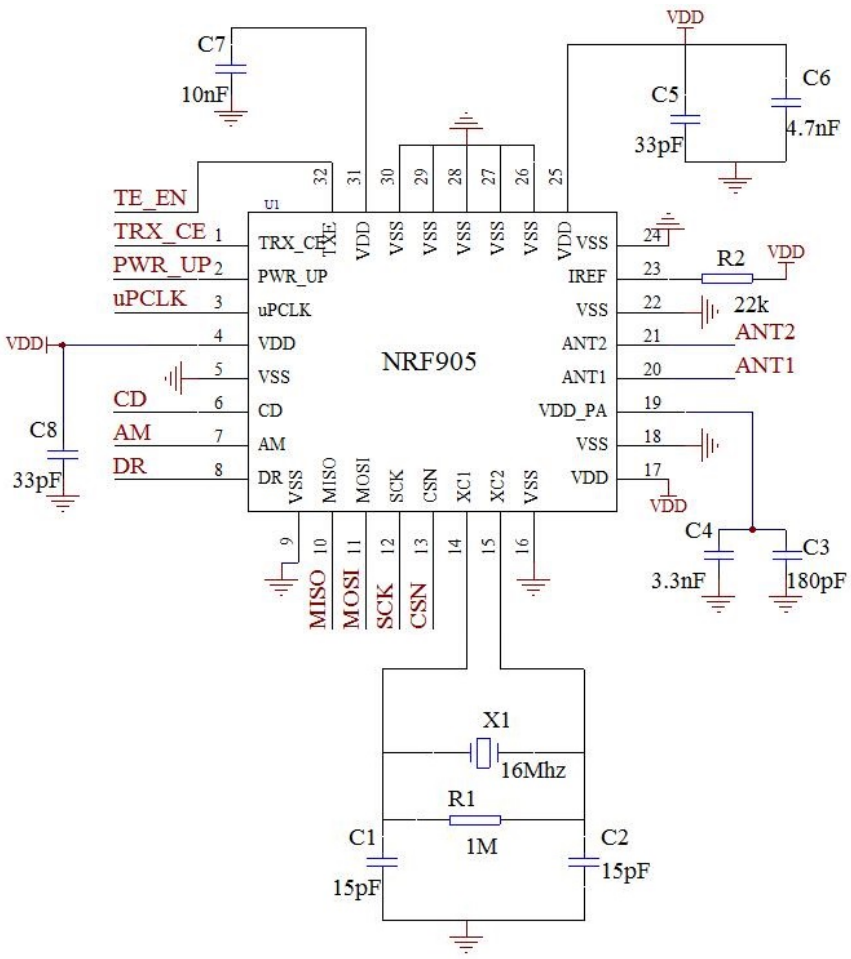

Fig. 5. nRF905 module

Instruction of configuration-register data is as follows: 0x4C: operating frequency is $430 \mathrm{MHz}$; 0x0C : receiving mode is established as normal mode; 0x44 : 4 bytes are used in the register which contains TX address, 4 bytes are used in the register which contains RX address ; 0x09: received valid data width; 0x11,0x22, 0x33, 0x44 : RX address; 0xDC : 16 bit CRC check, output clock frequency is $4 \mathrm{MHz}$.

\section{E. Control core}

MSP430F149 is used as control core in this system. It has different operation modes and five ultra-low power modes (CPU halts, external modules are still working).The CPU which incorporates hardware multiplier, large-capacity 16-bit registers, and $64 \mathrm{~KB}$ flash and $2 \mathrm{~KB}$ RAM guarantees the computational speed and data storage ${ }^{[1-4]}$.

\section{F. LCD display}

LCM0826A module is used to realize the displaying function. It is a liquid crystal display module with 8 sections, 4 serial interfaces. Its power-consumption is low: the typical current value in saving-power mode is smaller than $1 \mu \mathrm{A}$, and $50 \mu \mathrm{A}$ in the displaying mode. The contrast gradient is adjustable, the demonstration is clear, and the programming is simple.

\section{G. Analysis of power-consumption}

System's power-consumption is an important part of system performance appraisal. The chip and the module are usually at the sleeping state or the shutdown condition, and enter the active status after waken up. This may reduce the system power loss greatly. The operating current is shown in Table 1 according to the technical manual.

TABLE I. OPERATInG CURRENT

\begin{tabular}{|l|l|l|}
\hline & low-power mode & working mode \\
\hline MSP430 & $0.8 \mathrm{uA}$ & $560 \mathrm{uA}$ \\
\hline AD620 & -- & $0.9 \mathrm{~mA}$ \\
\hline SP3222E & $1 \mathrm{uA}$ & $1 \mathrm{~mA}$ \\
\hline nRF905 & $2.5 \mathrm{uA}$ & $12.5 \mathrm{~mA}$ \\
\hline LCM0826A & $1 \mathrm{uA}$ & $50 \mathrm{uA}$ \\
\hline SP481R & $1 \mathrm{uA}$ & $0.5 \mathrm{~mA}$ \\
\hline
\end{tabular}

III. THE TRANSPLANTATION IMPLEMENTATION OF UC/OS-II KERNEL

\section{A. Structure of $u C / O S-I I$}

It mainly consists of five parts: the core, the task processing, time processing, the task synchronization and correspondence, the CPU transplant and so on. The core(OSCore.c) is operating system's processing core, including the operating system initialization, the system operating, the leader of the interruption in and out, the clock tick, the task scheduler, the event processing and so on. The task processing part (OSTask.c): the content is related with duty operation closely, including the task establishment, the deletion, hanging up, restoring and so on. Operation such as the task delay is finished at the clock part(OSTime.c). Task synchronization and communication part: it's the event processing part, including the semaphores, mailbox, message queue, events flags and so on, mainly used for the interrelationship between tasks and services of accessing shared data. CPU interface parts: refers to the used transplant part of the CPU at uC/OS-II, mainly including the bottom realization of the interruption level task switching, the bottom realization of the task level task switching, production and processing of the clock tick, etc.

\section{B. Software design}

The essential part of software design includes: Operating system's transplant, memory allocation, user application procedure.

(1)As uC/OS-II has strong transplantable character, in the transplantable work, the code needed to modify is associated with the processor in the following files: OS_CUP.h, OS_CUP_A.s, OS_CPU_C.c. they contain settings of constants, definitions of macros and typedef, functions related with system task scheduling.

(2)Memory disposition and task management: Regarding the storage capacity, we should consider both $\mu \mathrm{C} / \mathrm{OS}-\mathrm{II}$ essence code and the application procedure code size. Each duty is an independent movement, provided with the independent stack space, and each duty's priority is different. Therefore when carrying on the software design, we should divide the system to certain duties, and distribute duties respective rational priority. $\mu \mathrm{C} / \mathrm{OS}-\mathrm{II}$ is responsible for task 
synchronization and communication, each duty uses semaphores, mailbox, message queue to carry on data sharing and task communication ${ }^{[5-7]}$.

(3)Application procedure design: according to priority order, the tasks are introduced as follows: data collection; Energy-consumption data storage; Liquid crystal display; Pressed key establishment; correspondence with the collection, after receiving the concentrator order, execution corresponding duty according to order request. Clock interruption which $\mu \mathrm{C} / \mathrm{OS}$-II provides is used to judge whether one duty is at the ready-state according to certain time-gap. The flow chart is shown in Fig. 6.

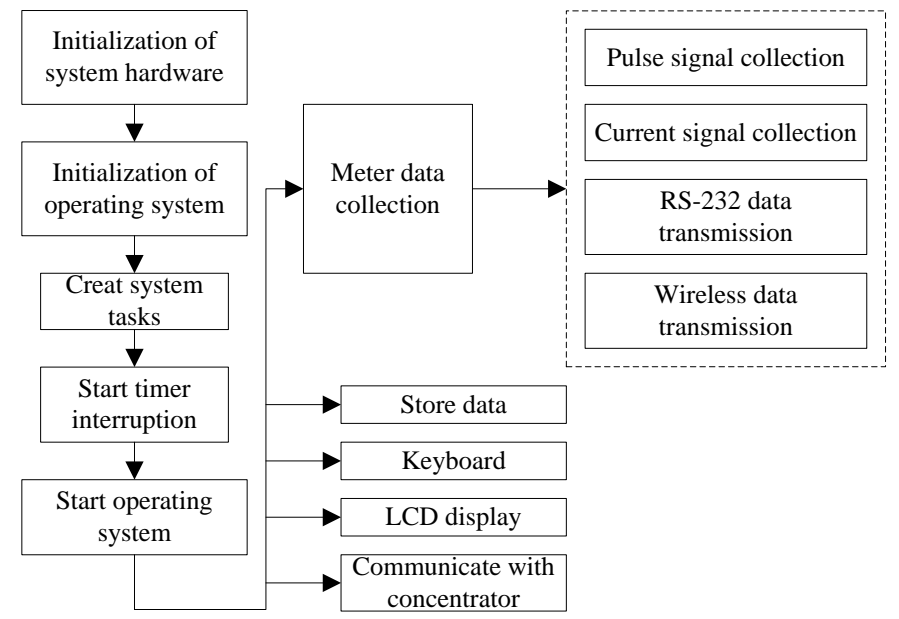

Fig. 6. Flow chart of software design

\section{Low power-consumption design}

In operating system, each task can preserve its own condition separately; therefore realizing low powerconsumption working mode is more nimble and convenient. This article has also carried on the design in the low power loss aspect: using $\mu \mathrm{C} / \mathrm{OS}$-II idle task expansion connection, to make the system enter some kind of low power-consumption working mode under the idling condition, to reduce the system power loss, (MSP430 interior Timer 0 is used as Clock Tick), periodically awakens CPU. $\mu \mathrm{C} / \mathrm{OS}-\mathrm{II}$ can manage 64 duties at most, and allocation a different priority for each task. Each duty may have five kinds of condition -- sleeping condition, the ready condition, the operating condition, the waiting condition and the interruption servicing condition. $\mu \mathrm{C} / \mathrm{OS}-\mathrm{II}$ belongs to the deprivable essence. It enters the task in ready state which has the highest priority. Once the high- priority task enters the ready condition, it may snatch CPU from the low-priority task. When $\mu \mathrm{C} / \mathrm{OS}-\mathrm{II}$ initialization, it will establish a lowest-priority task -- idle task. If no task enters ready condition, the idle task will start to work. The idle task will transfer function OSTaskIdleHook() which is the essence expanding connection for users. The idle task with nothing to do in fact is just an infinite loop waiting for interruption.
Therefore the user may use OSTaskIdleHook() to make CPU enter the low power-consumption mode ${ }^{[8]}$.

The inquiry in the program is replaced by the interruption, which makes CPU have nothing to do before the interruption, and enter the waiting or the sleeping mode. Reading RAM will bring a bigger power loss compared with reading Flash, therefore in software design the subroutine transfer should be reduced as far as possible and macro is used in place of the subroutine.

\section{CONCLUSION}

MSP430F149 micro-controller, whose function is complete, is advantageous for expansion. $\mu \mathrm{C} / \mathrm{OS}-\mathrm{II}$ operating system which is stable and reliable in practical application can save a lot of developing time, cut down costs. This design unifies MSP430 and $\mu \mathrm{C} / \mathrm{OS}-\mathrm{II}$, and has carried on low powerconsumption. The system which has many advantages such as small volume, convenient usage and high performance-toprice ratio, is used in the residential district energyconsumption data acquisition. It has a broad application prospect in the market.

\section{ACKNOWLEDGMENT}

This research was funded under the programs Anhui university natural science research project funding (KJ2016A156) and national natural science foundation of China (61071001).

\section{REFERENCES}

[1] Xu Jun, Dai Yawen, Zhang Yitao. “Design of Ultra-low Power Wireless Strain Sensor Based on MSP430”. Instrument Technique and Sensor. pp. 11-13, July 2008. (In Chinese)

[2] Zhen Liping, Si Shaowei. "Infrared Temperature Measurement System Based on MSP430”. 2011 Third International Conference on Measuring Technology and Mechatronics Automation. pp. 421-424, 2011.

[3] Bingjiang Gong. "Design of the intelligent heat meter based on MSP430FW425”, 2008 IEEE Conference on Cybernetics and Intelligent Systems, pp. 648-651, September 2008.

[4] Yanbing Zhang. "Design of Low-Power Wireless Communication System Based on MSP430 and nRF2401”, 2010 International Conference on Measuring Technology and Mechatronics Automation, pp. 1008-1010, March 2010.

[5] LIU Shuang, SHAN Ming-cai, KONG Xiang-jie, XUE Lin-yan, KOU Juan. "Development of Ultra-Low-Power Handheld Testing Instrument of Seismic Detector Based on $\mu \mathrm{C} / \mathrm{OS}-\mathrm{II}$ ”. Computer Information and Technology. pp. 224-225, April 2007. (In Chinese)

[6] Weili Zhou, Jingsheng Liao, Baopu Li, Jingrong Li. "A family medical monitoring system based on embedded uC/OS-II and GPRS”. 2012 IEEE International Conference on Information and Automation. pp. 663 $-667,2012$.

[7] Xibo Wang, Shan Jin, Zhongling Yang. "Several Methods of Design and Implementation to Solve Priority Inversion Problem in uC/OS-II”. 2013 6th International Conference on Intelligent Networks and Intelligent Systems (ICINIS). pp. 223 - 226, 2013.

[8] WEN Jing-xin, DONG Tao. "Design of Data Collection System Based on MSP430 and $\mu$ C/OS-II”. Fujian Computer. pp.100-101, February 2009. (In Chinese) 